\title{
Efficacy and Safety of Ranibizumab Intravitreal Injections versus Laser Photocoagulation in Patients with Diabetic Macular Edema
}

\author{
Fadia T. Ahmed ${ }^{1, *}$, Najah K.M. Al Quriashi ${ }^{2}$, Ibrahim A. Majeed $^{1}$ \\ ${ }^{1}$ Clinical Pharmacy Department, College of Pharmacy/ Baghdad University, Baghdad, Iraq \\ ${ }^{2}$ Ophthalmology Department, College of Medicine/ Baghdad University, Baghdad, Iraq \\ *Corresponding author: fadia81th@gmail.com
}

Received August 21, 2014; Revised August 27, 2014; Accepted September 01, 2014

\begin{abstract}
Objective: Diabetic macular edema (DME) is a swelling of the retina resulting from leakage of fluid from blood vessels within the macula (the centre of the retina), it involves retinal thickening and formation of hard exudates that occurs secondary to diabetic retinopathy. The aim of the study was to evaluate the safety and efficacy of ranibizumab versus laser therapy in DME patients. Methods: A randomized controlled open labeled clinical trial with 4 months duration was performed. 40 Patients with DME were randomly allocated to receive either ranibizumab $0.5 \mathrm{mg}$ intravitreal injection monthly for three months or laser photocoagulation at baseline and were evaluated at baseline and one month after completing their treatments. Clinical assessment was done by measuring best corrected visual acuity (BCVA), central retinal thickness (CRT), and monitoring of the adverse events. Results: Ranibizumab was superior to laser $(\mathrm{P}=0.0001)$ in improving $\mathrm{BCVA}$ letter score, mean change from baseline to the end of the study was $(+8.75 \mathrm{vs}+2.75)$, with significant increase in VA for both ranibizumab $(\mathrm{P}<0.0001)$ and laser $(\mathrm{P}=0.005)$. The mean CRT was significantly reduced from baseline with ranibizumab $(-126.4 \mu \mathrm{m})$ versus laser $(-$ $67.85 \mu \mathrm{m})$ both with significant change $(\mathrm{P}<0.0001)$. Ranibizumab was better in reducing CRT $(\mathrm{P}=0.0003)$. No endophthalmitis and no clinically significant increased intraocular pressure (IOP) for both ranibizumab and laser arms. One case of cataract was reported in laser arm. Other adverse events include eye pain, conjunctival hemorrhage (in both arms), increased lacrimation, foreign body sensation and eye floaters (in ranibizumab arm) and eye pruritus (in the laser arm). There were no systemic adverse events and no deaths reported. Conclusion: Ranibizumab provided significantly superior BCVA and CRT improvement over laser therapy. It had a good safety outcome similar to the established safety profile of ranibizumab. It was well tolerated in DME.
\end{abstract}

Keywords: ranibizumab, diabetic macular edema, laser photocoagulation

Cite This Article: Fadia T. Ahmed, Najah K.M. Al Quriashi, and Ibrahim A. Majeed, "Efficacy and Safety of Ranibizumab Intravitreal Injections versus Laser Photocoagulation in Patients with Diabetic Macular Edema." American Journal of Pharmacological Sciences, vol. 2, no. 4 (2014): 72-76. doi: 10.12691/ajps-2-4-3.

\section{Introduction}

Diabetic macular edema (DME) is a major cause of visual impairment in patients with diabetic retinopathy (DR) [1]. It can be defined as an advanced complication of DR that involves swelling of the macula (retinal centre) causing vision loss [4]. Currently laser photocoagulation (focal/grid) is the standard treatment for DME, it is effectively treated clinically significant macular edema (CSME), and reduced the risk of moderate vision loss by $50 \%$ at 3-year follow-up [3]. A 2-year study reported that focal/grid laser photocoagulation was more effective and had fewer side effects than intravitreal triamcinolone acetonide [4].

Frequency-doubled Nd:YAG (FD YAG) laser $(532 \mathrm{~nm}$ green) has the advantages of being efficiently absorbed in the retinal pigment epithelium with reduced risk of extensive neuro-retinal damage and less scattering that results in a more focused beam. It is also a solid-state device, portabile, efficient and reliabile [5].

Vascular endothelial growth factor (VEGF) levels are increased in the retina and vitreous of DR eyes [6]. It has been found to be extensively involved in the development and progression of DME [7]. VEGF induces neovascularization and microvascular leakage [8]. Thus, inhibiting VEGF can provide an alternative therapeutic modality in DME. Ranibizumab is an anti-VEGF antibody fragment (fully humanized monoclonal antibody fragment), that inhibits the activity of all active isoforms of VEGF [9]. It was approved by the FDA for the treatment of DME by intravitreal injection in 2012 [10]. It acts by decreasing the concentration of free VEGF in the vitreous and increasing the clearance of VEGF from the retina, thus decreasing its concentration in the retina [11].

Several studies investigated the efficacy and safety of ranibizumab in treating DME and compared it to laser photocoagulation. The RESOLVE study (phase II 
randomized multicenter) found that ranibizumab was significantly more effective than sham treatment (with rescue laser), it results in rapid and continuous improvements in best corrected visual acuity (BCVA) over 12 months and in reducing the central retinal thickness (CRT) (both $\mathrm{P}<0.0001$ ), and it was well tolerated [12]. The RESTORE study is a 12-month, double-masked, multicentered, lasercontrolled Phase III study which investigated the efficacy and safety of ranibizumab versus laser therapy for DME, it showed that treatment with ranibizumab both as monotherapy or combined treatment were significantly superior to laser monotherapy in improving BCVA and CRT reduction. Repeated intravitreal injection of ranibizumab was found to be safe and not associated with increased risk of cerebrovascular or cardiovascular events in DME patients over 12 months in the study [13].

The present study was designed assess the efficacy and safety of ranibizumab intravitreal injection in the treatment of Iraqi patients with visual impairment due to DME and to compare it with the conventional laser therapy.

\section{Subjects and Methods}

\subsection{Study Design}

The present study is a randomized controlled open labeled clinical trial with 4 months treatment and follow up periods was carried out over 8 months from October 2013 till June 2014 at Ophthalmology Department, Ghazy Al-Hariri Hospital for Specialized Surgeries at Medical City, and Dar Al-Salam Private Hospital. Informed consent was-obtained from all participants and ethical approval was obtained from the Ethics Committee by College of Pharmacy / Baghdad University. Patients were randomly allocated to receive either ranibizumab intravitreal injections $(0.5 \mathrm{mg})$ monthly for three months or undergo laser photocoagulation therapy at baseline. Ranibizumab was bought from Novartis pharma Company, Switzerland. A solid state $\mathrm{Nd}$ :YAG laser (532 nm), continuous wave, was used. A slit-lamp- laser delivery system and a fundus contact lens were used. Burns were produced using powers of between 70 and $210 \mathrm{~mW}$ pulse duration $50 \mathrm{~ms}$ and a spot size of $75 \mu \mathrm{m}$. Burns were applied in a scatter pattern on areas of retinal thickening and focused on microaneurysms. A single specialist surgeon (Dr. Najah K. M. Al Quraishi) delivered ranibizumab and laser treatments to all the patients in this study. Patients were evaluated at baseline and one month after completing their treatment.

\subsection{Sample Selection}

Forty adult patients (with type I or type II DM) had confirmed DME that not fully healed by previous treatment or newly diagnosed DME. And with stable DM treatment within 3 months before the study and expected to remain stable during the study. Exclusion criteria included patients with macular edema due to cause other than DM; previous ranibizumab or laser therapy in the last 3 months; cataract; glaucoma; external ocular infections; known hypersensitivity to ranibizumab; pregnancy; breast feeding; blood pressure >170/100; HbA1c > 11\%; history of chronic renal failure and arteriothrombotic events or other cardiac events in the last 6 months (including myocardial infarction, congestive heart failure, stroke or transient ischemic attack). Additionally, 20 healthy age and sex matched individuals to be considered as a control group.

\subsection{Clinical Evaluation}

Assessment of the patient's outcome was done using CRT measured by OCT and visual acuity measured as BCVA by snellen chart at baseline and one month after completing the treatment. And monitoring of the adverse events of ranibizumab and laser either by clinical examination for the objective data or by questionnaire for the subjective data. The conversion of Snellen visual acuity fractions to approximate Early Treatment Diabetic Retinopathy Study (approx ETDRS) letter scores was done for easier statistical analysis. The formula to convert Snellen visual acuity scores to approxETDRS letter scores is:

$$
\begin{aligned}
& \text { ApproxETDRS letter scores } \\
& =85+50 * \log (\text { Snellen decimal fraction })
\end{aligned}
$$

ApproxETDRS letter score may be rounded to the nearest letter [14].

\subsection{Statistical Analysis}

Statistical software (IBM SPSS version 20) was used for data input and analysis. Continuous variables were presented as mean \pm standard deviation (SD) and discrete variables were presented as numbers and frequencies. Chi square test for independence was used to test the significance of association between discrete variables. Continuous variables were tested by the Shapiro Wilk test to determine if they were normally or abnormally distributed. ANOVA test was used to test the significance of difference in the mean of 3 independent samples in normally distributed continuous variables. Unpaired t-test was used to test the significance of difference in the mean of two independent samples in normally distributed continuous variables and Mann Whitney test for abnormally distributed data. Findings with $\mathrm{P}$ value less than 0.05 were considered significant.

\section{Results}

\subsection{Efficacy}

Of a total of 51 patients who were randomized in this study, 40 completed the treatment (20 from the ranibizumab group and 20 from the laser group, Figure 1).

The mean age of ranibizumab group did not differ significantly from laser group and controls (54.85 \pm 4.01 years vs $54.30 \pm 4.49$ years vs $54.25 \pm 3.20$ years respectively, $\mathrm{P}>0.05$ ).

Also there was no statistical significant difference in the female to male ratio among ranibizumab group, laser group, and controls (9:11 (45\%) vs $11: 9$ (55\%) vs 12:8 $(60 \%)$ respectively, $\mathrm{P}>0.05$, Table 1$)$.

Baseline patients parameters showed that BCVA level was significantly lower in patients with DME than healthy control subjects $(\mathrm{P}<0.0001)$. Whereas CRT was significantly higher in patients with DME than those in control group ( $\mathrm{P}<0.0001$, Table 2$)$. 


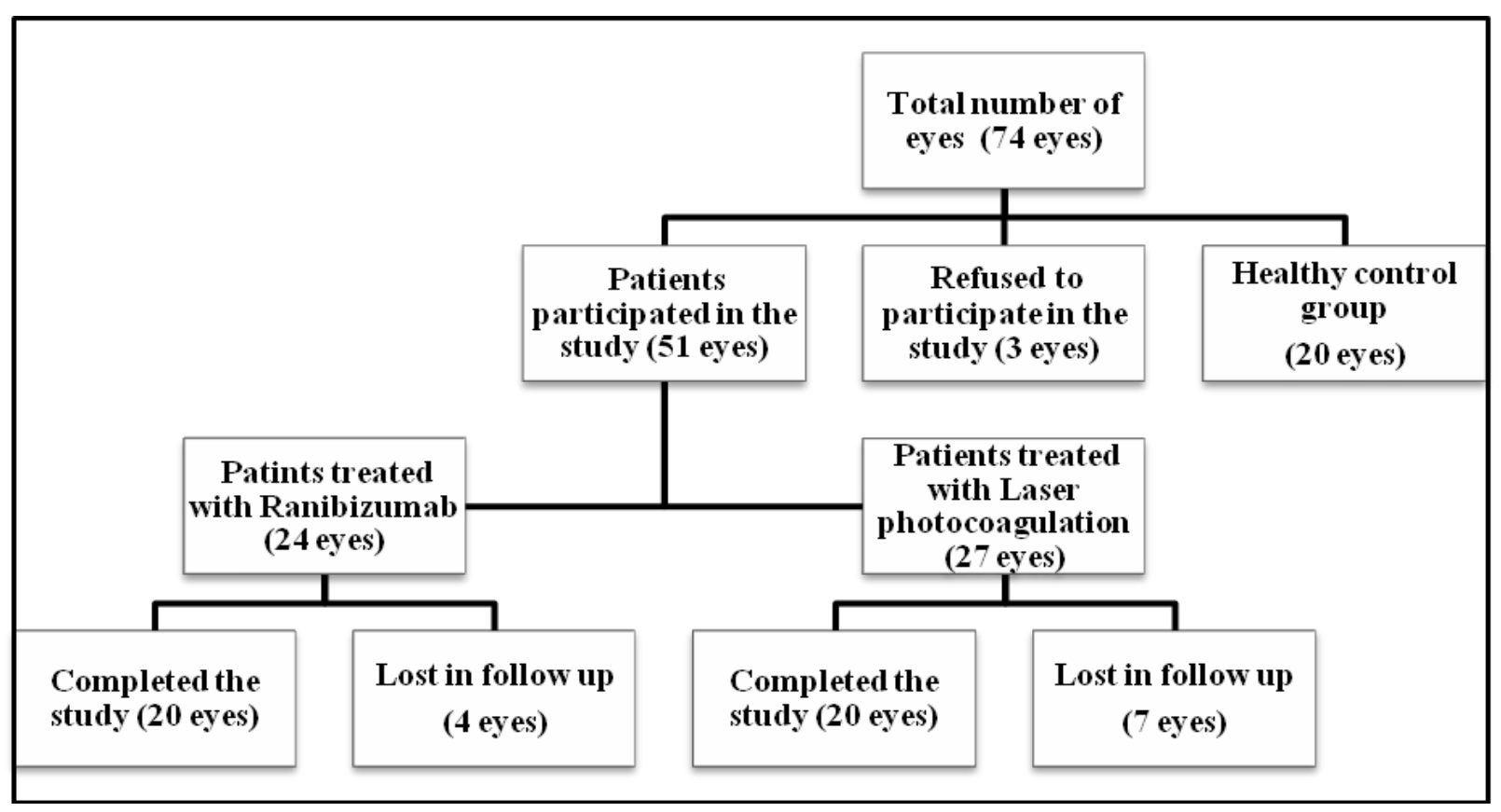

Figure 1. Total number of patients participated in the current study

Table 1. Baseline characteristics of patients and controls

\begin{tabular}{|c|c|c|c|c|}
\hline Parameter & $\begin{array}{c}\text { Ranibizumab } \\
(\mathrm{n}=20)\end{array}$ & $\begin{array}{c}\text { Laser } \\
(\mathrm{n}=20) \\
\end{array}$ & $\begin{array}{c}\text { Control } \\
(\mathrm{n}=20)\end{array}$ & P-value \\
\hline Age inyears (mean \pm SD) & $54.85 \pm 4.01$ & $54.30 \pm 4.49$ & $54.25 \pm 3.20$ & 0.867 \\
\hline Female: Male [n (\%)] & 9:11 (45\%) & $11: 9(55 \%)$ & $12: 8(60 \%)$ & 0.626 \\
\hline DM Type IDDM: NIDDM [n (\%)] & 1:19 (5\%) & $2: 18(10 \%)$ & $\cdots \cdots$ & 0.548 \\
\hline Duration of DM in years (mean \pm SD) & $13.85 \pm 3.64$ & $12.9 \pm 4.96$ & $\ldots .$. & 0.288 \\
\hline Family history of DM [n (\%)] & $14(70 \%)$ & $12(60 \%)$ & $\ldots \ldots$ & 0.507 \\
\hline HbA1c (mean\% \pm SD \%) & $7.22 \% \pm 0.59 \%$ & $7.05 \% \pm 0.53 \%$ & $\ldots .$. & 0.227 \\
\hline DM Treatment with Insulin [n (\%)] & $10(50 \%)$ & $8(40 \%)$ & $\ldots .$. & 0.525 \\
\hline Hypertension [n (\%)] & $12(60 \%)$ & $9(45 \%)$ & $7(35 \%)$ & 0.280 \\
\hline Dyslipidemia [n (\%)] & $2(10 \%)$ & $3(15 \%)$ & $1(5 \%)$ & 0.574 \\
\hline Smoking [n (\%)] & $4: 16(20 \%)$ & $8: 12(40 \%)$ & $6: 14(30 \%)$ & 0.386 \\
\hline
\end{tabular}

Continuous variables presented as Mean \pm Standard deviation; and discrete variables as numbers and frequencies.

Table 2. Baseline parameters of the patients and controls

\begin{tabular}{|c|c|c|c|}
\hline Parameter & $\begin{array}{c}\text { Ranibizumab } \\
(\mathrm{n}=20)\end{array}$ & $\begin{array}{c}\text { Laser } \\
(\mathrm{n}=20)\end{array}$ & $\begin{array}{c}\text { Control } \\
(\mathrm{n}=20)\end{array}$ \\
\hline Best corrected visual acuity (BCVA) & $53.5 \pm 12.98$ & $60.25 \pm 11.05$ & $80.25 \pm 6.17$ \\
\hline Central retinal thickness (CRT) & $392 \pm 61.95$ & $362 \pm 27.77$ & $196.8 \pm 7.45$ \\
\hline
\end{tabular}

Continuous variables presented as Mean \pm Standard deviation.

After 4 months of starting ranibizumab treatment and 1 month of laser photocoagulation, we found a highly significant increase in BCVA for both ranibizumab and laser groups $(\mathrm{P}<0.0001, \mathrm{P}=0.005$ respectively) with ranibizumab being superior to laser $(\mathrm{P}=0.0001)$.

The mean change \pm SD in BCVA letter score from baseline to the end of the study (4 months for ranibizumab arm and 1 month of laser arm) was 8.75 \pm 4.83 and 2.75 \pm 3.43 approxETDRS letters respectively. The proportion of patients who gained $\geq 10$ BCVA letters was greater in ranibizumab group than in laser group (60\% vs $10 \%)$ respectively.

There was a significant decrease in CRT for both groups $(\mathrm{P}<0.0001)$, ranibizumab was found to be more effective than laser in decreasing CRT ( $\mathrm{P}=0.0003$, Table 3$)$. The mean change \pm SD in CRT from baseline to the end of the study was $-126.4 \pm 58.87 \mu \mathrm{m}$ and $-67.85 \pm 13.37 \mu \mathrm{m}$ respectively. At the end of the study, there was a greater proportion of patients with CRT $<275 \mu \mathrm{m}$ in ranibizumab arm than in the laser arm (45\% vs $20 \%$ ) respectively (Table 3 ).

Table 3. Changes in the parameters at the end of the study

\begin{tabular}{|c|c|c|}
\hline Parameter & $\begin{array}{c}\text { Ranibizumab } \\
(\mathrm{n}=20)\end{array}$ & $\begin{array}{c}\text { Laser } \\
(\mathrm{n}=20)\end{array}$ \\
\hline Best corrected visual acuity (BCVA) & $8.75 \pm 4.83$ & $2.75 \pm 3.43$ \\
\hline Central retinal thickness (CRT) & $-126.4 \pm 58.87$ & $-67.85 \pm 13.37$ \\
\hline
\end{tabular}

Continuous variables presented as Mean \pm Standard deviation. 


\subsection{Safety}

The present study showed no serious ocular adverse events (increased intraocular pressure (IOP) and cataract) were reported in the ranibizumab arm, whereas there was one case of cataract development in the laser arm that was suspected not to be related to the study procedure. There were no reported cases of endophthalmitis in any of the treatment arms.

The most frequent ocular adverse event in ranibizumab and laser arms was eye pain (25\% vs 35\%, respectively) followed by conjunctival hemorrhage (20\% vs 5\%, respectively). Other ocular adverse events include increased lacrimation, foreign body sensation and eye floaters (20\%, $5 \%$ and $10 \%$ respectively) that were reported in ranibizumab arm only, while in the laser arm, there was $10 \%$ reported incidence of eye pruritus (Table 4).

Table 4. Most common ocular adverse events
\begin{tabular}{|c|c|c|}
\hline Adverse event & $\begin{array}{c}\text { Ranibizumab } \\
(\mathrm{n}=20)\end{array}$ & $\begin{array}{c}\text { Laser } \\
(\mathrm{n}=20)\end{array}$ \\
\hline Eye pain [n (\%)] & $5(25 \%)$ & $7(35 \%)$ \\
\hline Conjunctival hemorrhage [n (\%)] & $4(20 \%)$ & $1(5 \%)$ \\
\hline Increased lacrimation [n (\%)] & $4(20 \%)$ & 0 \\
\hline Foreign body sensation [n (\%)] & $1(5 \%)$ & 0 \\
\hline Eye floaters [n (\%)] & $2(10 \%)$ & 0 \\
\hline Eye pruritus [n (\%)] & 0 & $2(10 \%)$ \\
\hline
\end{tabular}

Data presented as number of reported cases and percents.

There was no clinically significant difference between ranibizumab and laser arms for mean IOP, pain incidence and conjunctival hemorrhage incidence. There were no systemic adverse events and no deaths reported during the study.

\section{Discussion}

Visual acuity is the most common assessment of visual function, because it can be performed easily with simple equipment. It considered as a "gold standard" for visual outcomes in clinical trials [15].

The current study demonstrates that both ranibizumab and laser increased BCVA significantly. Overall ranibizumab was significantly superior to laser in improving BCVA in patients with visual impairment due to DME with greater proportion of patients gained $\geq 10$ BCVA letters in ranibizumab group compared to laser group.

Optical coherence tomography (OCT) that used to measure CRT has many advantages including being a noninvasive device that obtains cross-sectional, high resolution images of the retina and thus may detect retinal thickening [16]. It is considered as objective and sensitive assessment of macular edema [17].

In the present study, there was a significant decrease in CRT in both ranibizumab and laser groups. Ranibizumab produced a greater reduction in CRT than that produced by laser. There was a significant difference between the effect of ranibizumab and laser on CRT. The proportion of patients with $\mathrm{CRT}<275 \mu \mathrm{m}$ was greater in ranibizumab arm than in the laser arm.

These efficacy results are consistent with results of the RESOLVE study which concluded that ranibizumab resulted in significant and continuous improvement in
BCVA and CRT over a period of 12 months compared with sham treatment. The mean change in BCVA letter score from baseline to month $12,+7.8$ for ranibizumab vs -0.1 for sham; $\mathrm{P}<0.0001$ ) [12].

The LUCIDATE study (a prospective, randomized, single-masked clinical trial) which assessed the functional and structural effects of ranibizumab versus macular laser therapy in patients with DME demonstrates that ranibizumab monotherapy results in larger improvements in visual function and reduction in central retinal thickness than laser treatment. The mean change in BCVA letter score in ranibizumab arm was +6.0 vs -0.9 letters lost for laser arm. There was a significant improvement in BCVA in the ranibizumab arm but there was a non significant result in the laser arm $(\mathrm{P}=0.083)$ with mean deterioration in visual acuity [18].

The difference in the laser efficacy may be due to the difference in the baseline visual acuity of the participating patients, the subjects in the ranibizumab arm had a mean BCVA that was 7 letters more than that of the laser arm in LUCIDATE study while in this study the laser arm mean BCVA is 6.75 letters more than ranibizumab arm. This may have a limiting effect on their potential for improvement because of the ceiling effect inherent with visual acuity testing. Also the difference in baseline BCVA is a limitation to the study because the two groups differ in the severity of the disease and as a result differ in their responses to the investigations done [18]. Or may be attributed to the difference in the follow up period, in our study the follow up for laser patients was after one month while for LICIDATE study the follow up was first done after three months.

The present study results were consistent with the RESTORE study [13] and the REVEAL study [19] (which is a multicentered phase III study similar to RESTORE, compared the efficacy and safety of $0.5 \mathrm{mg}$ ranibizumab as monotherapy or combined with laser versus laser monotherapy in Asian patients with DME). The mean reduction in the BCVA letter score from baseline was significantly superior with ranibizumab $(\mathrm{P}<0.0001)$ than laser treatment in both studies. The mean CRT for ranibizumab monotherapy or combined groups were also significantly lower compared with the laser group in both RESTORE and REVEAL studies $(\mathrm{P}=0.002$ and $\mathrm{P}=0.001$ respectively).

There was a numerical difference in the BCVA and CRT between those studies and the present study which may be attributed to the difference in the baseline characteristics of the participated patients. Additionally, these studies had different study designs and retreatment criteria, also the short duration of this study in comparison to the above studies.

In terms of safety, ranibizumab intravitreal injections were well tolerated in patients with visual impairment due to DME. There were no serious ocular adverse events observed in ranibizumab arm.

There were no cases of increased IOP, no cases of endophthalmitis in both of the treatment arms and one case of cataract development in the laser arm that was suspected not to be related to the study procedure. There were no systemic adverse events and no deaths in the study. Other ocular adverse events were observed but with no significant difference between ranibizumab and laser arms. 
The current safety findings are consistent with the results of other studies used ranibizumab in treating DME.

The safety results from three clinical trials RESOLVE [12], RESTORE [13] and DRCR.net [20] demonstrated that ranibizumab is effective and well tolerated in the treatment of DME. These reports may lead to DME treatment shifting from laser to the new approach of using ranibizumab [13].

In conclusion, ranibizumab provided significantly superior BCVA and CRT improvement over the standard laser therapy. It had a good safety outcome that is similar to the established safety profile of ranibizumab. It was well tolerated in treating patients with visual impairment due to DME.

However, future longer clinical trials are required with longer follow up periods to confirm the long term efficacy and safety of ranibizumab.

\section{References}

[1] Klein R, Knudtson MD, Lee KE, et al. The Wisconsin Epidemiologic Study of Diabetic Retinopathy XXIII: the twentyfive- year incidence of macular edema in persons with type 1 diabetes. Ophthalmology 2009; 116: 497-503.

[2] Johnson MW. Etiology and treatment of macular edema. Am J Ophthalmol 2009; 147: 11-21.

[3] Early Treatment Diabetic Retinopathy Study Research Group, Photocoagulation for diabetic macular edema. Early Treatment Diabetic Retinopathy Study report number 1. Arch Ophthalmol 1985; 103: 1796-1806.

[4] Diabetic Retinopathy Clinical Research Network. A randomized trial comparing intravitreal triamcinolone acetonide and focal/grid photocoagulation for diabetic macular edema. Ophthalmology 2008; 115: 1447-1449.

[5] M. Saeed, D. Parmar, D. Mchugh. Frequency-doubled Nd:YAG laser for the treatment of exudative diabetic maculopathy. Eye 2001; 15: 712-718.

[6] Aiello LP, Avery RL, Arrigg PG, et al. Vascular endothelial growth factor in ocular fluid of patients with diabetic retinopathy and other retinal disorders. N Engl J Med 1994; 331: 1480-1487.

[7] Aiello LP, Bursell SE, Clermont A, et al. Vascular endothelial growth factor-induced retinal permeability is mediated by protein kinase C in vivo and suppressed by an orally effective betaisoform-selective inhibitor. Diabetes 1997; 46: 1473-1480.

[8] Poulaki V. Hypoxia in the pathogenesis of retinal disease. In Retinal Vascular Disease. Joussen AM, Gardner TW, Kirchhof B, Ryan SJ, Eds. Berlin, Germany, Springer, 2007, 121-138.

[9] Ferrara N, Damico L, Shams N, et al. Development of ranibizumab, an anti-vascular endothelial growth factor antigen binding fragment, as therapy for neovascular age-related macular degeneration. Retina 2006; 26: 859-70.

[10] Jain A, Varshney N, Smith C. The Evolving treatment options for diabetic macular edema. International Journal of Inflammation 2013; Vol 2013: 1-10.

[11] Stefa'nsson E. Diabetic macular edema. Saudi Journal of Ophthalmology 2009; 23: 143-148.

[12] Massin P, Bandello F, Garweg J, et al. Safety and efficacy of ranibizumab in diabetic macular edema (RESOLVE study): a 12 month, randomized, controlled, doublemasked, multicenter phase II study. Diabetes Care 2010; 33: 2399-405.

[13] Mitchell P, Bandello F, Schmidt-Erfurth U, et al. The RESTORE study: ranibizumab monotherapy or combined with laser versus laser monotherapy for diabetic macular edema. Ophthalmology. 2011; 118(4): 615-625.

[14] Gregori NZ, Feuer W, Rosenfeld PJ. Novel method for analyzing snellen visual acuity measurements. Retina 2010; Jul-Aug; 30(7): 1046-1050.

[15] Peter K. Kaiser MD. Prospective evaluation of visual acuity assessment: A comparison of snellen versus ETDRS charts in clinical practice (an AOS thesis). Trans Am Ophthalmol Soc 2009; 107: 311-324.

[16] Huang D, Swanson EA, Lin CP, et al: Optical coherence tomography. Science 1991; 254: 1178-81.

[17] Bressler M.N, Edwards R.A, ANDREW N. Retinal thickness on stratus optical coherence tomography in people with diabetes and minimal or no diabetic retinopathy. American Journal of Ophthalmology 2008; May; 145(5):894-901.

[18] Comyn O, Sivaprasad S, Peto T. A randomized trial to assess functional and structural effects of ranibizumab versus laser in diabetic macular edema (the LUCIDATE study). Am J Ophthalmol 2014; 157: 960-970.

[19] Ohji M, Ishibashi T; REVEAL study group. Efficacy and safety of ranibizumab $0.5 \mathrm{mg}$ as monotherapy or adjunctive to laser versus laser monotherapy in Asian patients with visual impairment due to diabetic macular edema: 12-month results of the REVEAL study. Invest Ophthalmol Vis Sci. 2012; 53: ARVO E-abstract 4664.

[20] Diabetic Retinopathy Clinical Research Network, Elman MJ, Aiello LP, Beck RW, et al. Randomized trial evaluating ranibizumab plus prompt or deferred laser or triamcinolone plus prompt laser for diabetic macular edema. Ophthalmology 2010; 117: 1064-77. 